Original Research Article

\title{
A neuropathic pain component as a predictor of improvement in pain interference after radiotherapy for painful tumors: A secondary analysis of a prospective observational study
}

\author{
Tetsuo Saito ${ }^{\mathrm{a}, \mathrm{b}, *}$, Etsushi Tomitaka ${ }^{\mathrm{c}}$, Ryo Toya ${ }^{\mathrm{a}}$, Tomohiko Matsuyama ${ }^{\mathrm{a}}$, Satoshi Ninomura ${ }^{\mathrm{a}}$, \\ Takahiro Watakabe ${ }^{\mathrm{a}}$, Natsuo Oya ${ }^{\mathrm{a}}$ \\ a Department of Radiation Oncology, Kumamoto University Hospital, Kumamoto, Japan \\ ${ }^{\mathrm{b}}$ Department of Radiation Oncology, Hitoyoshi Medical Center, Hitoyoshi, Japan \\ ${ }^{\mathrm{c}}$ Department of Radiation Oncology, Kumamoto Medical Center, Kumamoto, Japan
}

\section{A R T I C L E I N F O}

\section{Article history:}

Received 1 August 2018

Accepted 9 August 2018

Available online 13 August 2018

\section{Keywords:}

Palliative radiotherapy

Neuropathic pain

Painful tumors

Pain interference

\begin{abstract}
A B S T R A C T
Background and purpose: We previously demonstrated that patients with a tumor-related neuropathic pain component were more likely to experience a pain response after radiotherapy (RT) than those without. It is unknown whether the presence of a neuropathic component also favorably influences pain interference. In a secondary analysis of our previous prospective observational study, we investigated if the presence of a neuropathic component of the index pain caused by the irradiated tumors predicts greater reduction in pain interference.

Material and methods: For patients scheduled for RT for painful tumors, Brief Pain Inventory data were collected at initiation of RT and 1, 2, and 3 months thereafter. Multivariable linear regression analyses were performed to investigate the effects of the presence of a neuropathic component on the changes in pain interference scores (i.e., follow-up minus baseline). We used 10 covariates as potential confounders.

Results: Of the 302 analyzable patients, 93 (31\%) were diagnosed as having a neuropathic component of the index pain. Multivariable linear regression analyses revealed that all the point estimates of regression coefficients at 1-, 2-, and 3-month follow-up were negative values; some were statistically significant. At 2-month follow-up, patients with a neuropathic component experienced greater reductions in their pain interference scores for walking ability $(p=0.048)$, normal work $(p=0.021)$, sleep $(p=0.001)$, and enjoyment of life $(p=0.010)$ than those without it.

Conclusions: The presence of a neuropathic pain component predicted a greater reduction in pain interference after RT. Patients with neuropathic tumor-related pain should be offered the option of receiving palliative RT.
\end{abstract}

(C) 2018 The Authors. Published by Elsevier B.V. on behalf of European Society for Radiotherapy and

Oncology. This is an open access article under the CC BY-NC-ND license (http://creativecommons.org/ licenses/by-nc-nd/4.0/)
Neuropathic pain occurs in $19 \%$ to $39 \%$ of cancer patients [1-3]; it may be challenging therapeutically and have a substantial impact on patients' quality of life [4]. Neuropathic cancer pain may be directly caused by tumors or be treatment-related. Although pharmacotherapy is the mainstay of neuropathic pain management [3], it is important not to miss the opportunity to

\footnotetext{
* Corresponding author at: Department of Radiation Oncology, Kumamoto University Hospital, 1-1-1, Honjo, Chuo-ku, Kumamoto-shi, Kumamoto 860-8556, Japan.

E-mail addresses: tsaito@kumamoto-u.ac.jp (T. Saito), tomitaka@kumamed.jp (E. Tomitaka), ryo108@kumamoto-u.ac.jp (R. Toya), matsutomo_llp@yahoo.co.jp (T. Matsuyama), n-oya@kumamoto-u.ac.jp (N. Oya).
}

reverse the cause of the pain with appropriate oncological management, including radiotherapy (RT) [4]. A few studies have investigated the effects of RT on neuropathic tumor-related pain and have demonstrated that it can effectively palliate this type of pain $[5,6]$.

When selecting patients to receive palliative RT for painful tumors, it is important to predict which patients would benefit from this treatment. In our previous study, we demonstrated that patients with a neuropathic component of the index pain caused by the irradiated tumors were more likely to experience a pain response after RT than those without it [7]. It is not known whether the presence of a neuropathic component also favorably influences pain interference. When assessing interventions for pain, reduced 
interference in daily activity rather than a simple reduction in pain intensity is a relevant endpoint that reflects the true benefits for patients [8]. Therefore, in a secondary analysis of our previous prospective observational study, we investigated if the presence of a neuropathic component of the index pain predicts a greater reduction in pain interference after RT for painful tumors.

\section{Material and methods}

\section{Patients and study design}

The present study is a secondary analysis of our previously published prospective observational study that was conducted at three medical centers [7]. In the primary study, we analyzed 302 patients (enrolled between July 2013 and September 2017) who were scheduled to receive RT for painful tumors (Fig. 1); we evaluated the characteristics of the patients, their tumors, and their pain to identify the predictors of pain palliation after RT [7]. The data of these 302 patients were used in this secondary study to investigate the effect of the presence of a neuropathic component on the change in pain interference scores after RT. This secondary study was approved by the participating centers' institutional review boards; written informed consent was obtained from all participants for the primary study.

\section{Evaluation}

We previously reported how the patients were assessed at baseline and follow-up evaluations [7]. In brief, immediately prior to RT, the treating radiation oncologist identified the pain caused by the irradiated tumor using physical examination and diagnostic imaging; this pain was recorded as the index pain for the study. The treating radiation oncologist recorded whether the index pain had a neuropathic component according to the definition provided by the International Association for the Study of Pain - Neuropathic Pain Special Interest Group [9]. Patients with definite and probable neuropathic pain were recorded as having a neuropathic component. The Brief Pain Inventory (BPI) short form (Japanese version) was used to evaluate the intensity of pain and its interference in the patient's life using an 11-point scale (0 to 10); higher scores indicate greater pain intensity and interference [10]. Patients assessed their worst pain (in terms of the index pain) experienced in the previous 3 days. Pain interference was assessed using seven subscales: general activity, mood, walking ability, normal work, relations with other people, sleep, and enjoyment of life. The BPI data and analgesic data were collected at baseline, and 1, 2, and 3 months ( \pm 7 days) after initiation of RT.

\section{Statistical analysis}

The patients' characteristics, analgesic use, and baseline pain interference scores were analyzed using the Mann-Whitney U test for continuous variables; the Fisher exact test was used for categorical variables. Univariable and multivariable linear regression analyses were performed to investigate the effects of the presence of a neuropathic component of the index pain on the change in pain interference scores. The outcome variables were the changes in the functional interference scores from baseline (i.e., follow-up minus baseline). In the multivariable analysis, we used 10 covariates as potential confounders: age, sex, Eastern Cooperative Oncology

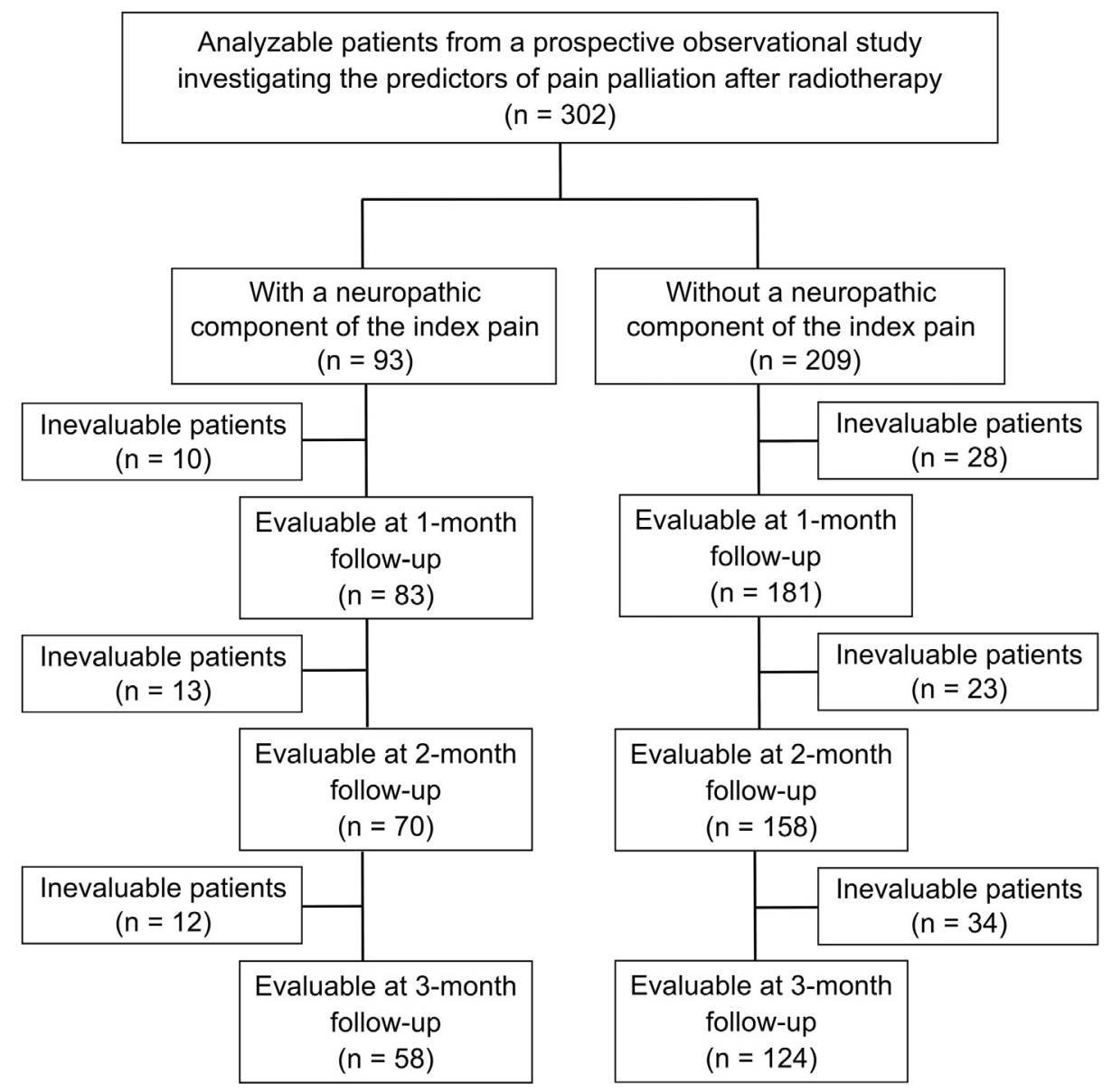

Fig. 1. Flow diagram of the study cohort. The index pain is the pain caused by the irradiated tumor. 
Group (ECOG) performance status, hematologic tumor, tumor involvement of the bone, worst pain score at baseline, opioid analgesic use at baseline, adjuvant analgesic use at baseline, palliative intent of the RT, and total radiation dose. The ECOG performance status ( $\leq 1 \mathrm{vs.}>1$ ) and worst pain score at baseline ( $\leq 7 \mathrm{vs} .>7)$ were treated as binary variables; age and total radiation dose were treated as continuous variables. Normality of residuals was assessed by inspection of Q-Q plots and histograms. The variance inflation factor was used to detect multicollinearity between independent variables. All tests were two-tailed; $p<0.05$ was considered statistically significant. We did not adjust for multiple comparisons in this exploratory study. All statistical analyses were performed using SPSS software, version 24 (IBM SPSS, Armonk, NY).

\section{Results}

\section{Patients}

Of the 302 analyzable patients, 93 (31\%) were diagnosed as having a neuropathic component of the index pain (definite, 52 patients; probable, 41 patients; Fig. 1); 83 (89\%) of the 93 patients also had nociceptive pain caused by the index tumors treated with RT. Patients with a neuropathic component had greater pain intensity and worse ECOG performance status than patients without (Table 1); they also reported significantly worse baseline pain interference scores for general activity, walking ability, relations with other people, and sleep (Table 2). There was no significant difference in the total radiation dose between the patients with a neuropathic component and those without (Table 1).

\section{Analgesic use}

At baseline, the patients with a neuropathic component were using opioid analgesics more frequently than those without the component (Table 3). At 1-month follow-up, patients with a neuropathic component were more frequently using opioid and adjuvant analgesics than those without. At 2- and 3-month follow-up, there was no significant difference in analgesic use between the two patient groups. At baseline and 1-, 2-, and 3-month follow-up, the median daily oral morphine equivalent doses were 23,23 , 15 , and $19 \mathrm{mg}$, respectively, in patients with a neuropathic component; the doses were $0,8,0$, and $0 \mathrm{mg}$, respectively, in the patients without a neuropathic component.

Pain

The mean worst pain scores at baseline and 1-, 2-, and 3-month follow-up are presented in Fig. 2. At baseline and 1-, 2-, and 3month follow-up, the median worst pain scores (in terms of the index pain) were $8,3,2$, and 2 , respectively, in the patients with a neuropathic component; these scores were $7,2,1$, and 1 , respectively, in the patients without a neuropathic component.

\section{BPI pain interference scores}

The mean pain interference scores at baseline and 1-, 2-, and 3month follow-up are presented in Fig. 2. For the patients with a neuropathic component, the median differences in scores (i.e., follow-up minus baseline) at 1-, 2-, and 3-month follow-up were

Table 1

Baseline patient characteristics $(n=302)$.

\begin{tabular}{|c|c|c|c|c|c|}
\hline \multirow[t]{2}{*}{ Characteristic } & \multicolumn{2}{|c|}{$\begin{array}{l}\text { Neuropathic component } \\
(\mathrm{n}=93)\end{array}$} & \multicolumn{2}{|c|}{$\begin{array}{l}\text { No neuropathic component } \\
(\mathrm{n}=209)\end{array}$} & \multirow[t]{2}{*}{$p$} \\
\hline & No. & $\%$ & No. & $\%$ & \\
\hline Age, years & & & & & 0.003 \\
\hline Median & 70 & & 65 & & \\
\hline Range & $35-91$ & & 21-89 & & \\
\hline Sex & & & & & 0.033 \\
\hline Female & 33 & 35 & 103 & 49 & \\
\hline Male & 60 & 65 & 106 & 51 & \\
\hline ECOG performance status & & & & & 0.023 \\
\hline 0 & 11 & 12 & 52 & 25 & \\
\hline 1 & 36 & 39 & 83 & 40 & \\
\hline 2 & 25 & 27 & 51 & 24 & \\
\hline 3,4 & 21 & 23 & 23 & 11 & \\
\hline Irradiated tumor & & & & & 0.36 \\
\hline Solid tumor & 78 & 84 & 184 & 88 & \\
\hline Hematologic tumor & 15 & 16 & 25 & 12 & \\
\hline Bone involvement by the tumor & & & & & $<0.001$ \\
\hline No & 8 & 9 & 86 & 41 & \\
\hline Yes & 85 & 91 & 123 & 59 & \\
\hline Worst pain score at baseline & & & & & 0.006 \\
\hline $1-2$ & 2 & 2 & 8 & 4 & \\
\hline $3-4$ & 8 & 9 & 53 & 25 & \\
\hline $5-7$ & 32 & 34 & 70 & 33 & \\
\hline $8-10$ & 51 & 55 & 78 & 37 & \\
\hline Intent of radiation therapy & & & & & 0.035 \\
\hline Curative & 13 & 14 & 52 & 25 & \\
\hline Palliative & 80 & 86 & 157 & 75 & \\
\hline Total radiation dose, Gy & & & & & 0.93 \\
\hline Median & 30 & & 30 & & \\
\hline Range & $8-62$ & & $6-70.4$ & & \\
\hline$\leq 20$ & 17 & 18 & 59 & 28 & \\
\hline $20-30$ & 43 & 46 & 60 & 29 & \\
\hline $30-45$ & 17 & 18 & 32 & 15 & \\
\hline$>45$ & 16 & 17 & 58 & 28 & \\
\hline
\end{tabular}


Table 2

Baseline pain interference scores from the Brief Pain Inventory.

\begin{tabular}{|c|c|c|c|c|c|c|c|}
\hline \multirow[t]{2}{*}{ Item } & \multicolumn{3}{|c|}{ Neuropathic component } & \multicolumn{3}{|c|}{ No neuropathic component } & \multirow[t]{2}{*}{$p^{*}$} \\
\hline & No. & Median & IQR & No. & Median & IQR & \\
\hline General activity & 91 & 6 & 3 to 10 & 204 & 5 & 2 to 8 & 0.037 \\
\hline Mood & 93 & 6 & 2 to 8 & 203 & 5 & 2 to 8 & 0.12 \\
\hline Walking ability & 93 & 5 & 2 to 9 & 204 & 4 & 1 to 8 & 0.030 \\
\hline Normal work & 91 & 6 & 3 to 10 & 201 & 5 & 1.5 to 9 & 0.065 \\
\hline Relations with other people & 92 & 4.5 & 1 to 8 & 204 & 2.5 & 0 to 6 & 0.040 \\
\hline Sleep & 92 & 5 & 2 to 8 & 205 & 3 & 1 to 6 & 0.016 \\
\hline Enjoyment of life & 92 & 5.5 & 2 to 10 & 202 & 5 & 1 to 9 & 0.099 \\
\hline
\end{tabular}

Abbreviation: $\mathrm{IQR}=$ interquartile range.

"Mann-Whitney U test.

Table 3

Analgesic use.

\begin{tabular}{|c|c|c|c|c|c|}
\hline \multirow[t]{2}{*}{ Analgesic use } & \multicolumn{2}{|c|}{ Neuropathic component } & \multicolumn{2}{|c|}{ No neuropathic component } & \multirow[t]{2}{*}{$p^{*}$} \\
\hline & No. & $\%$ & No. & $\%$ & \\
\hline Baseline & $\mathrm{n}=93$ & & $\mathrm{n}=209$ & & \\
\hline Opioid analgesic use & 64 & 69 & 101 & 48 & 0.001 \\
\hline Adjuvant analgesic use & 38 & 41 & 67 & 32 & 0.15 \\
\hline 1-month follow-up & $\mathrm{n}=83$ & & $\mathrm{n}=181$ & & \\
\hline Opioid analgesic use & 63 & 76 & 94 & 52 & $<0.001$ \\
\hline Adjuvant analgesic use & 47 & 57 & 75 & 41 & 0.024 \\
\hline 2-month follow-up & $\mathrm{n}=70$ & & $\mathrm{n}=158$ & & \\
\hline Opioid analgesic use & 41 & 59 & 76 & 48 & 0.15 \\
\hline Adjuvant analgesic use & 37 & 53 & 62 & 39 & 0.061 \\
\hline 3-month follow-up & $\mathrm{n}=58$ & & $\mathrm{n}=124$ & & \\
\hline Opioid analgesic use & 33 & 57 & 56 & 45 & 0.16 \\
\hline Adjuvant analgesic use & 28 & 48 & 49 & 40 & 0.33 \\
\hline
\end{tabular}

Fisher exact test.

as follows: $-3,-3$, and -4 for general activity; $-2.5,-3$, and -4 for mood; $-2,-3$, and -2 for walking ability; $-2.5,-3$, and -2 for normal work; $-2,-1$, and -1 for relations with other people; $-2,-3$, and -3 for sleep; and $-2,-3$, and -3 for enjoyment of life, respectively. The median differences in scores for the patients without a neuropathic component at 1-, 2-, and 3-month followup were as follows: $-2,-2$, and -2 for general activity; $-2,-2$, and -2 for mood; $0,-1$, and -1 for walking ability; $-2,-1.5$, and -1 for normal work; $-1,-1$, and 0 for relations with other people; $-1,-1$, and -1 for sleep; and $-1.5,-1$, and -2 for enjoyment of life, respectively.

Effect of the presence of a neuropathic pain component on the changes in pain interference scores

There were no missing values in the 10 covariates. Inspection of the $\mathrm{Q}-\mathrm{Q}$ plots and histograms revealed that the assumption of normality of residuals was not violated. The variance inflation factors ranged from 1.11 to 3.08 , which indicated that there was no multicollinearity problem. The univariable and multivariable linear regression analyses demonstrated that the patients with a neuropathic component of the index pain tended to experience greater reduction in their pain interference scores than those without this pain component (Table 4). All the point estimates of regression coefficients at 1-, 2-, and 3-month follow-up were negative values, and some were statistically significant. Greater differences in the pain interference scores between the two groups were seen at 2and 3-month follow-up than at 1-month follow-up.

\section{Discussion}

Our present study demonstrated that patients that had a neuropathic component of the index pain tended to experience greater reductions of their pain interference scores after RT than those without this pain component. We also observed that, at baseline, patients with a neuropathic component had greater pain intensity, worse ECOG performance status, and worse pain interference scores than patients without it, despite their more frequent use of opioid analgesics.

We found that the reduction of the pain interference scores after RT tended to be greater among patients with a neuropathic component. Our linear regression analyses demonstrated that greater differences in the pain interference scores between the two groups (i.e., those with and without neuropathic pain) were seen at 2-month follow-up than at 1-month follow-up. While more frequent analgesic use was observed in the patients having a neuropathic component at 1-month follow-up, no significant difference in analgesic use was observed between the two groups at 2month follow-up. The difference in analgesic use between the two groups decreased between the 1- and 2-month follow-up evaluations; however, the differences in pain interference scores increased during this period. This increase in the difference in functional interference between the two groups may be due to the effects of RT.

In our previous study, which is the largest to date to examine the effects of neuropathic pain on the pain response to RT, patients with a neuropathic component were more likely to experience a pain response after RT [7]. In contrast, other smaller studies did not find significant differences in the pain response rates between patients with and without neuropathic pain $[11,12]$. In the present study, we observed that the presence of a neuropathic component predicts a greater improvement in pain interference. These findings reinforce the benefits that patients with neuropathic tumorrelated pain may derive from RT.

A previous clinical study demonstrated that normalization of sensory abnormality after RT predicts greater improvement in pain 

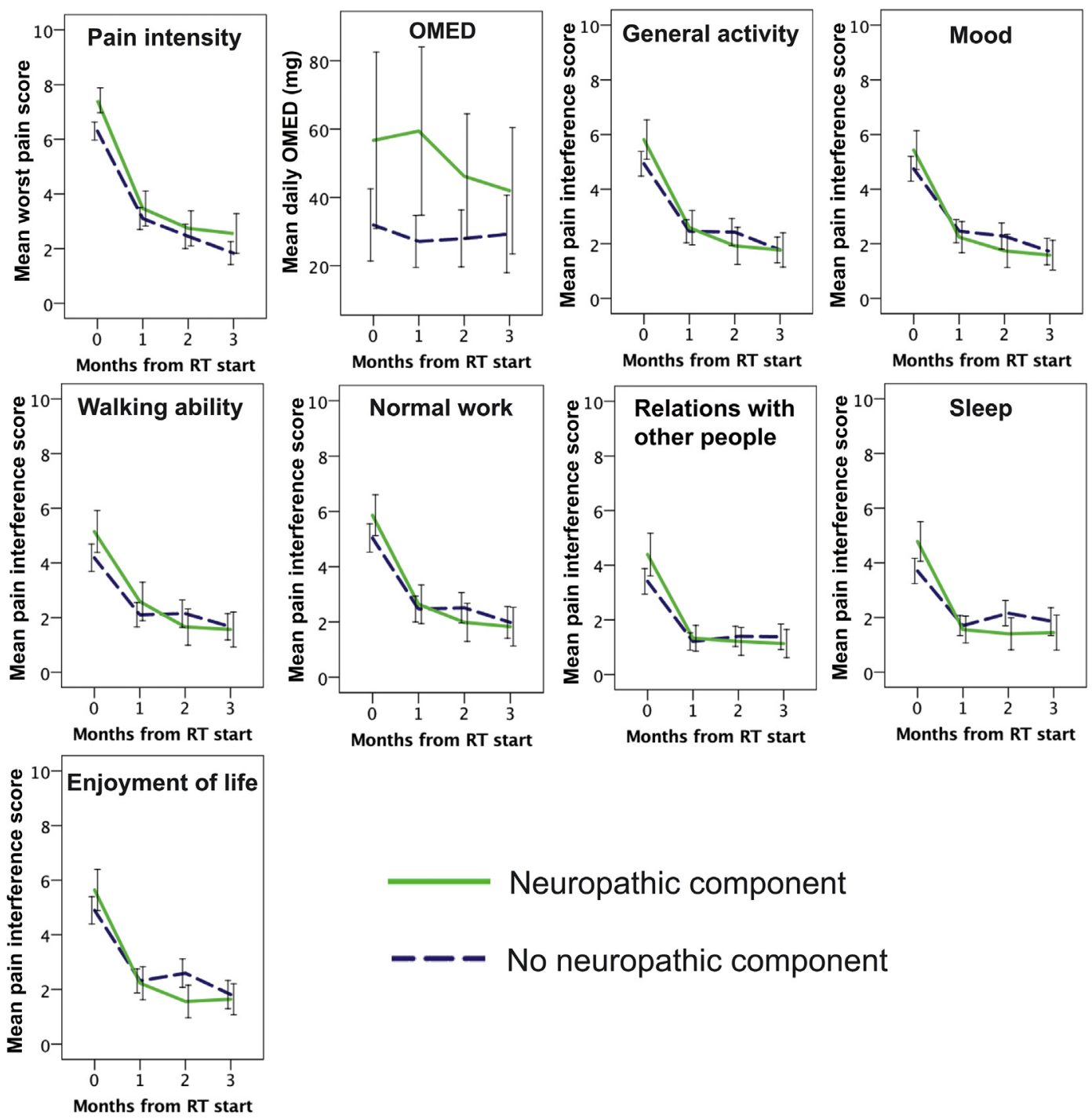

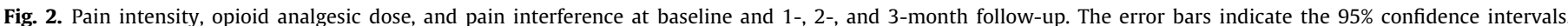
Abbreviations: $\mathrm{RT}=$ radiotherapy; OMED = oral morphine equivalent dose

Table 4

Linear regression models to investigate the effect of the presence of a neuropathic pain component on the changes in pain interference scores from baseline.

\begin{tabular}{|c|c|c|c|c|c|c|c|c|c|c|c|c|}
\hline \multirow[t]{2}{*}{ Item } & \multicolumn{4}{|c|}{ 1-month follow-up } & \multicolumn{4}{|c|}{ 2-month follow-up } & \multicolumn{4}{|c|}{ 3-month follow-up } \\
\hline & No. & $\beta$ & $95 \% \mathrm{CI}$ & $p$ & No. & $\beta$ & $95 \% \mathrm{CI}$ & $p$ & No. & $\beta$ & $95 \% \mathrm{CI}$ & $p$ \\
\hline \multicolumn{13}{|l|}{ Univariable } \\
\hline General activity & 253 & -0.63 & -1.60 to 0.35 & 0.21 & 210 & -1.24 & -2.40 to -0.09 & 0.036 & 168 & -1.32 & -2.60 to -0.04 & 0.044 \\
\hline Mood & 252 & -0.89 & -1.88 to 0.09 & 0.075 & 208 & -0.87 & -2.05 to 0.32 & 0.15 & 166 & -1.32 & -2.65 to 0.01 & 0.052 \\
\hline Walking ability & 253 & -0.36 & -1.35 to 0.63 & 0.47 & 212 & -1.35 & -2.54 to -0.16 & 0.026 & 166 & -1.44 & -2.70 to -0.18 & 0.026 \\
\hline Normal work & 241 & -0.67 & -1.78 to 0.45 & 0.24 & 204 & -1.32 & -2.57 to -0.07 & 0.039 & 166 & -1.07 & -2.41 to 0.28 & 0.12 \\
\hline Relations with other people & 253 & -0.71 & -1.64 to 0.22 & 0.14 & 209 & -0.95 & -2.04 to 0.14 & 0.086 & 166 & -1.14 & -2.33 to 0.05 & 0.060 \\
\hline Sleep & 255 & -1.17 & -2.14 to -0.20 & 0.018 & 212 & -1.74 & -2.87 to -0.61 & 0.003 & 167 & -1.63 & -2.93 to -0.33 & 0.014 \\
\hline Enjoyment of life & 250 & -0.79 & -1.83 to 0.26 & 0.14 & 205 & -1.62 & -2.90 to -0.35 & 0.013 & 165 & -1.08 & -2.48 to 0.32 & 0.13 \\
\hline \multicolumn{13}{|l|}{ Multivariable $^{*}$} \\
\hline General activity & 253 & -0.48 & -1.51 to 0.55 & 0.36 & 210 & -1.31 & -2.64 to 0.03 & 0.054 & 168 & -1.24 & -2.69 to 0.20 & 0.092 \\
\hline Mood & 252 & -0.89 & -1.96 to 0.18 & 0.10 & 208 & -1.18 & -2.53 to 0.18 & 0.087 & 166 & -1.25 & -2.81 to 0.31 & 0.12 \\
\hline Walking ability & 253 & -0.49 & -1.51 to 0.53 & 0.35 & 212 & -1.33 & -2.65 to -0.01 & 0.048 & 166 & -1.48 & -2.81 to -0.15 & 0.030 \\
\hline Normal work & 241 & -0.39 & -1.57 to 0.79 & 0.52 & 204 & -1.64 & -3.03 to -0.25 & 0.021 & 166 & -1.16 & -2.64 to 0.32 & 0.12 \\
\hline Relations with other people & 253 & -0.38 & -1.37 to 0.61 & 0.45 & 209 & -0.43 & -1.66 to 0.80 & 0.49 & 166 & -0.58 & -1.93 to 0.76 & 0.39 \\
\hline Sleep & 255 & -1.27 & -2.33 to -0.21 & 0.019 & 212 & -2.27 & -3.56 to -0.98 & 0.001 & 167 & -1.58 & -3.13 to -0.03 & 0.046 \\
\hline Enjoyment of life & 250 & -0.66 & -1.77 to 0.45 & 0.24 & 205 & -1.92 & -3.37 to -0.47 & 0.010 & 165 & -0.66 & -2.26 to 0.94 & 0.42 \\
\hline
\end{tabular}

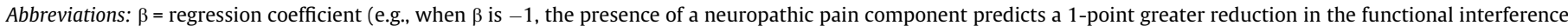
score from baseline); $\mathrm{CI}=$ confidence interval.

Outcome variables are the changes in the functional interference scores from baseline (i.e., follow-up minus baseline).

"The covariates are age, sex, Eastern Cooperative Oncology Group performance status, hematologic tumor, tumor involvement of the bone, worst pain score at baseline, opioid analgesic use at baseline, adjuvant analgesic use at baseline, palliative intent of the radiation therapy, and total radiation dose. 
intensity [13]. The patients with bone metastases that experienced normalization of abnormal warm sensation on the skin over the area of cancer-induced pain had greater pain score reduction [13]. The authors of this previous study reported that alterations in specific sensory characteristics seem to be associated with an increased likelihood of successful analgesia from palliative RT [13]. They demonstrated which patients responded best using Quantitative Sensory Testing after RT. In contrast, we demonstrated which patients benefited most from RT by diagnosing the presence or absence of a neuropathic component before RT. Thus, our results will enable better patient selection for palliative RT.

In addition, we found that, at baseline, patients with a neuropathic component of the index pain had greater pain intensity, worse performance status, worse pain interference scores, and used opioid analgesics more frequently than patients without this component. Our findings are consistent with past studies that investigated neuropathic cancer pain, which demonstrated that patients with neuropathic pain were likely to have a higher pain intensity, worse performance status, worse pain interference, worse quality of life, and more frequent analgesic use [14-16]. Neuropathic cancer pain is associated with a negative impact on patients' life quality and functioning despite greater use of analgesics. Palliative RT may be a good intervention for this type of pain, considering its favorable influence on pain interference that was demonstrated in this study.

Diagnosis of a neuropathic component was performed by the treating radiation oncologists in the present study. Making a diagnosis based on the definition provided by the International Association for the Study of Pain [9] requires an experienced physician. We are uncertain if the results of our study will be reproducible in other medical facilities; thus this uncertainty in generalizability may be a limitation of our study. However, one advantage of our study is that the treating radiation oncologists diagnosed whether the neuropathic component was related to the index tumor that was scheduled to receive RT. Although screening questionnaires to diagnose neuropathic pain $[17,18]$ can be used reproducibly at any facility, it is not certain if the neuropathic pain identified by use of the questionnaires is caused by the irradiated tumors. Additionally, these screening questionnaires are often insufficiently accurate $[1,12,19]$. Neuropathic cancer pain can have many causes other than tumors. When attempting to predict the effects of RT, it is necessary to know if the neuropathic pain component is caused by the index tumors treated with RT.

In conclusion, a secondary analysis of our previous prospective observational study demonstrated that the presence of a neuropathic component of the index pain predicted a greater reduction in pain interference after RT for painful tumors. This observation supports our previous findings that patients with a neuropathic component were more likely to experience a pain response after RT. Patients with neuropathic tumor-related pain should be offered the option of receiving palliative RT.

\section{Sources of support}

The authors have no sources of support to report.

\section{Conflict of interest}

The authors have no conflicts of interest to declare.

\section{References}

[1] Morgan KJ, Anghelescu DL. A review of adult and pediatric neuropathic pain assessment tools. Clin J Pain 2017;33:844-52.

[2] Roberto A, Deandrea S, Greco MT, Corli O, Negri E, Pizzuto M, et al. Prevalence of neuropathic pain in cancer patients: pooled estimates from a systematic review of published literature and results from a survey conducted in 50 Italian palliative care centers. J Pain Symptom Manage 2016;51(1091-102):e4.

[3] Vadalouca A, Raptis E, Moka E, Zis P, Sykioti P, Siafaka I. Pharmacological treatment of neuropathic cancer pain: a comprehensive review of the current literature. Pain Pract 2012;12:219-51.

[4] Fallon MT. Neuropathic pain in cancer. Br J Anaesth 2013;111:105-11.

[5] Roos DE, Turner SL, O'Brien PC, Smith JG, Spry NA, Burmeister BH, et al. Randomized trial of $8 \mathrm{~Gy}$ in 1 versus $20 \mathrm{~Gy}$ in 5 fractions of radiotherapy for neuropathic pain due to bone metastases (Trans-Tasman Radiation Oncology Group, TROG 96.05). Radiother Oncol 2005;75:54-63.

[6] Roos DE. Radiotherapy for neuropathic pain due to bone metastases. Ann Palliat Med 2015;4:220-4.

[7] Saito T, Toya R, Tomitaka E, Matsuyama T, Ninomura S, Oya N. Predictors of pain palliation after radiation therapy for painful tumors: a prospective observational study. Int J Radiat Oncol Biol Phys 2018:101:1061-8.

[8] Bennett MI. Mechanism-based cancer-pain therapy. Pain 2017;158(Suppl 1): S74-8.

[9] Treede RD, Jensen TS, Campbell JN, Cruccu G, Dostrovsky JO, Griffin JW, et al. Neuropathic pain: redefinition and a grading system for clinical and research purposes. Neurology 2008;70:1630-5.

[10] Cleeland CS, Ryan KM. Pain assessment: global use of the Brief Pain Inventory. Ann Acad Med Singapore 1994:23:129-38.

[11] Nakamura N, Takahashi O, Zenda S, Kawamori J, Ogita M, Onozawa M, et al. Neuropathic pain features in patients with bone metastases. Clin Oncol (R Coll Radiol) 2016;28:204-8.

[12] MacLeod N, Kelly C, Stobo J, McMahon L, Taggart D, Fallon M, et al. Pain in malignant pleural mesothelioma: a prospective characterization study. Pain Med 2016;17:2119-26.

[13] Scott AC, McConnell S, Laird B, Colvin L, Fallon M. Quantitative Sensory Testing to assess the sensory characteristics of cancer-induced bone pain after radiotherapy and potential clinical biomarkers of response. Eur J Pain 2012;16:123-33.

[14] Arthur J, Tanco K, Haider A, Maligi C, Park M, Liu D, et al. Assessing the prognostic features of a pain classification system in advanced cancer patients. Support Care Cancer 2017;25:2863-9.

[15] Oh SY, Shin SW, Koh SJ, Bae SB, Chang H, Kim JH, et al. Multicenter, crosssectional observational study of the impact of neuropathic pain on quality of life in cancer patients. Support Care Cancer 2017;25:3759-67.

[16] Rayment C, Hjermstad MJ, Aass N, Kaasa S, Caraceni A, Strasser F, et al. Neuropathic cancer pain: prevalence, severity, analgesics and impact from the European Palliative Care Research Collaborative-Computerised Symptom Assessment study. Palliat Med 2013;27:714-21.

[17] Bennett MI, Smith BH, Torrance N, Potter J. The S-LANSS score for identifying pain of predominantly neuropathic origin: validation for use in clinical and postal research. J Pain 2005;6:149-58.

[18] Bouhassira D, Attal N, Alchaar H, Boureau F, Brochet B, Bruxelle J, et al. Comparison of pain syndromes associated with nervous or somatic lesions and development of a new neuropathic pain diagnostic questionnaire (DN4). Pain 2005;114:29-36.

[19] Mathieson S, Maher CG, Terwee CB, Folly de Campos T, Lin CW. Neuropathic pain screening questionnaires have limited measurement properties. A systematic review. J Clin Epidemiol 2015;68:957-66. 